\title{
Comparing the influence of sunspot activity and geomagnetic activity on winter surface climate
}

I. Roy ${ }^{1,2}$, T. Asikainen ${ }^{2}$, V. Maliniemi², K. Mursula²

${ }^{1}$ University of Exeter, U.K.

${ }^{2}$ Space Climate Research unit, University of Oulu, Finland 


\section{Abstract.}

We compare here the effect of geomagnetic activity (using the aa index) and sunspot activity on surface climate using sea level pressure dataset from Hadley centre during northern winter. Previous studies using the multiple linear regression method have been limited to using sunspots as a solar activity predictor. Sunspots and total solar irradiance indicate a robust positive influence around the Aleutian Low. This is valid up to a lag of one year. However, geomagnetic activity yields a positive NAM pattern at high to polar latitudes and a positive signal around Azores High pressure region. Interestingly, while there is a positive signal around Azores High for a 2-year lag in sunspots, the strongest signal in this region is found for aa index at 1-year lag. There is also a weak but significant negative signature present around central Pacific for both sunspots and aa index. The combined influence of geomagnetic activity and Quasi Biannual Oscillation (QBO $30 \mathrm{hPa}$ ) produces a particularly strong response at mid to polar latitudes, much stronger than the combined influence of sunspots and QBO, which was mostly studied in previous studies so far. This signal is robust and insensitive to the selected time period during the last century. Our results provide a useful way for improving the prediction of winter weather at middle to high latitudes of the northern hemisphere.

Key words: geomagnetic activity, sunspot activity, ENSO, QBO, Sea level pressure. 


\section{Introduction.}

The energy driving the global atmosphere mainly comes from the Sun. The Earth is subject to the effects of solar activity, which varies according to the solar 11-year cycle and in longer trends. The extent to which the changes in solar activity affect climate has been the subject of considerable interest over many years. The detected solar signals have been found to be regionally different and also seasonally dependent.

Observational studies have suggested a significant solar related impact on sea surface temperatures in the tropical Pacific (van Loon and Meehl, 2007, Meehl et al. 2008), tropical circulations (Haigh et al. 2005, Meehl et al. 2008), climatological precipitation maxima in the tropics (Van Loon et al, 2004), Northern Hemisphere winter blocking (Barriopedro et al. 2008; Lockwood et al. 2010), North Atlantic Oscillation (NAO) (Lockwood et al. 2010, Maliniemi et al. 2013, 2014), Northern Annular Mode (NAM) (Ogi et al. 2004) and Antarctic polar vortex (Haigh and Roscoe, 2009).

Christoforou and Hameed (1997) showed that during sunspot maxima the Centre of Action (COA) of the Aleutian Low (AL) pressure system moves north-westward (the movement to west by as much as $700 \mathrm{~km}$ ), while during sunspot minima, it moves in the reverse direction. Shifts in the COA change storm trajectories and cause large anomalies in regional climate. Apart from varying position, $A L$ even exhibits significant changes in intensity; there is an average $1.6 \mathrm{mb}$ difference between years of high and low solar activity. Using the Multiple Linear Regression (MLR) technique, Roy and Haigh (2010) detected a very strong solar signal on Sea Level Pressure (SLP) in winter (DJF) around the Aleutian Low, with a secondary opposite signal in the tropical Pacific. Signal around Aleutian Low is robust and insensitive to varying the time period (Roy and Haigh, 2012). Van Loon et al. (2007), using solar peak year compositing, also noticed a similar signal around AL although their study only focused on peak years rather that high solar activity years as considered in MLR technique.

Using the method of solar peak year compositing of nearly 150 years of data, Van Loon et al. (2007) and Meehl et al. (2008) showed that for increased solar forcing, there is a cold event like pattern in the Pacific during DJF. Van Loon and Meehl (2007), using NOAA SST datasets over the time period 1871-1989, detected a very strong tropical solar signal, resembling that of the negative phase of ENSO. However, Roy and Haigh (2010) and Tung and Zhou (2010), using different techniques and considering longer time records, were 
unable to detect any clear solar signal around tropical Pacific. Those studies were based on high solar years rather than only peak years.

An underlying quasi-decadal variability in the inter-annual ENSO is detected in several studies (White et al., 2008; Zhang et al., 1997; Zhao et al., 2003; Chen et al. 2004; Hegyi and Deng 2011). Extra-tropical forcing theory of ENSO (Kao and Yu 2009, Yu et al. 2010, Yu and Kim 2011) suggests ENSO could be excited by forcing from extra-tropics and then grow by the equatorial ocean advection. McPhaden (2011) on the other hand suggested that the recent decadal change in ENSO could be partly due to natural variability.

Studies indicate that during the Northern Hemisphere winter, it is necessary to group the meteorological data according to the phase of QBO, in order to find a clear signal of the 11-year solar cycle in the stratosphere. For example, Labitzke and van Loon (1992), using data for the years 1956 -1991, showed that warm polar temperatures tend to occur during the west phase of the QBO at solar maximum and east phase at solar minimum. Baldwin and Dunkerton (2005) showed that fluctuations in the strength of the stratospheric polar vortex are coupled downward with surface climate. Large-amplitude variations in the strength of the stratospheric polar vortex are typically followed, with a lag of less than one month, by same signed anomalies in the tropospheric circulation that persist for up to 2 months. Following Labitzke and van Loon (1992) and Baldwin and Dunkerton (2005), Haigh and Roscoe (2006) constructed a new Solar ${ }^{\star}$ QBO index in their analysis to study polar annular modes in both hemispheres. This index was also used recently by Roy and Haigh (2011) and Roy (2013) to study surface signature due to various combinations of the Sun and QBO.

Although a solar signal is evident in many climate parameters, as also discussed in details by Gray et al. (2010), and it is obvious that the Sun drives the climate of Earth, the exact physical mechanisms by which solar variations manifest themselves in the climate are yet poorly understood. One reason is that it is hard to isolate each possible solar driver, e.g., UV, Total solar irradiance (TSI), geomagnetic activity, solar cosmic or magnetospheric energetic particle precipitation, and to pinpoint it to a single climate response. Moreover, which one is a better predictor for specific surface climate variability is still an area of major debate.

TSI follows the variable sunspot activity and has influence on climate through radiative forcing. The direct impact is found on sea surface temperatures and hydrological cycle (Lean and Rind, 2001). Enhanced solar UV is responsible for heating the upper and middle atmosphere which can dynamically couple down to troposphere (Kodera et al. 2002, Haigh et al. 2005). Solar UV variability is also strongly correlated with sunspot variability. Solar 
$\mathrm{F} 10.7 \mathrm{~cm}$ radio flux, which can be measured by ground based instruments is often used as a long term proxy of solar UV or sunspot variability.

Geomagnetic activity is another solar related driver (actually solar wind related driver) that has been found to have influence on surface climate (Bochnıcek and Hejda, 2005; Bochnicek et al. 2012; Maliniemi et al., 2014). It has two separate components, one due to coronal mass ejections (CME) and the other due to high speed solar wind streams (HSS). CME's vary roughly in phase with the sunspot cycle, whereas the HSS have a variable lag with respect to the sunspot cycle and a peak in the declining phase of the sunspot cycle.

Several recent studies have shown a connection between the Northern Hemisphere winter conditions with solar UV flux (Ineson, 2011) or geomagnetic activity (Bochnıcek et al., 2012; Maliniemi et al., 2014). Those indicated a positive correlation with the NAO index in recent decades (Thejll et al. 2003), causing significant variation in surface temperature on the northern hemisphere (Rozanov et al. 2005; Seppälä et al. 2009; Baumgaertner et al. 2011). Strengthening of the stratospheric polar vortex by geomagnetic activity was found to be stronger during high solar activity (Seppälä et al. 2013). It was also shown that both sunspot activity and geomagnetic activity modulate the NAO pattern, but the effect of geomagnetic activity is stronger (Bochnıcek and Hejda, 2005). Bochnıcek et al. (2012) showed that during high solar activity of recent decades, the geopotential height anomalies in the troposphere that resemble the positive NAO pattern came mainly from geomagnetic activity. Li et al. (2011) proposed that the relation between geomagnetic activity and the NAO is nonlinear and nonstationary. It is negative for weak to medium activity period, while positive during high phase, and it also depends on multidecadal variation in solar cycles. Woollings et al. (2010) showed that there is a consistent and stronger connection between the open solar flux and NAO than between solar F10.7 cm radio flux and NAO. (Open solar flux is the magnetic flux that enters the heliosphere from the top of the solar atmosphere, the solar corona). Lockwood et al. (2010) associated timings of minimum open solar flux with cold winters in Europe and blocking events.

Energetic particles are responsible, e.g., for the ionisation of the upper and middle atmosphere, and have a major influence on composition and temperature. Energetic particles are known to be important for magnetosphere-ionosphere-thermosphere coupling. Moreover, through NOx production they can even impact the lower polar stratosphere, which is a potential route to modulate NAO. Recent study by Maliniemi et al. (2013) showed that energetic electron precipitation (EEP) correlates significantly well with winter surface temperature resembling that of positive NAO pattern. A proposed mechanism linking these drivers to winter circulation variability at polar regions involves 
energetic particle precipitation into the high-latitude atmosphere causing significant changes in atmospheric chemistry, e.g., by producing nitrogen oxides (NOx) in the upper atmosphere (Seppälä et al. 2007; Sinnhuber et al. 2011). NOx in turn can descend down (Funke et al. 2005) and affect ozone balance in the stratosphere during polar winter, when NOx lifetimes are long due to the absence of sunlight and a large-scale downward motion exists in the polar atmosphere (Randall et al. 2005). This mechanism is also well observed in the chemistry-climate models (Rozanov et al. 2005; Baumgaertner et al. 2011).

Apart from direct solar or solar wind related drivers, galactic cosmic rays (GCR) have also been suggested to influence the climate of the Earth. We however note that sunspots and GCR are strongly anti-correlated and sunspots can also be used as an effective reverse proxy for GCR for its climate influence.

Due to the close relation between sunspots and the various solar radiation related drivers (TSI and UV flux), it is difficult to isolate their individual influences to surface climate. As noted above, the same is also true for GCR. On the other hand, the solar cycle evolution of geomagnetic activity, solar wind speed, and EEP has been different from sunspot cycle at least during the space age, peaking in the declining phase of the sunspot cycle. As discussed above, several studies found better correlation between surface winter climate and geomagnetic activity than sunspots (or TSI or UV activity) (Maliniemi et al., 2014, Bochnıcek et al., 2012). Hence comparative analyses are needed to better explore those relations. This is the focus of our current study.

In the current analysis, we compare the effect of geomagnetic activity and sunspot activity on surface climate during northern winter. Section 2 presents our methodology and data, whereas Section 3 presents and discusses our results. Discussion mainly focuses on SLP during northern winter. First part of Section 3 considers the differences and similarities between signatures of the aa index and sunspot number (SSN). It also compares results around the Aleutian Low and discusses controversies relating to sunspots and ENSO. Second part of Section 3 compares the combined influence of QBO and aa index with QBO and SSN on SLP. Conclusions are given in Section 4.

\section{Methodology and Data}

The method used here is the Multiple Linear Regression analysis with AR (1) noise model. Multiple linear regression can be represented as follows: 


$$
Y=\overline{\bar{X}} \bar{\beta}+\bar{u}
$$

Here $\mathbf{Y}$ is a vector of rank $\mathrm{n}$ containing the time series of the climate data to be explained. $X$ is a matrix, comprising the time series of $m$ indices, which are thought to explain the data, $\beta$ is a vector of rank $m$ that contains coefficients of the explaining indices and $\mathbf{u}$ is the noise term which is unobserved and may arise due to various sources (e.g., internal noise, observational errors, un-modelled variability etc.). Here the variability due to various climate factors is assumed to follow autoregressive noise model of order one (AR(1)). Using a noise model of higher order does not make much difference to the results. Finally, the level of confidence in the values of $\beta$ derived for each index is estimated using Student's t-test.

In this methodology, noise coefficients are calculated simultaneously with the components of variability so that the residual is consistent with a red noise model of order one. First the autocorrelation and variance of the noise are estimated from the residual ( $\mathbf{Y}-\mathbf{b} \mathbf{X}$, where $\mathbf{b}$ is an estimate of $\beta$ ); then a red noise function of order one is fitted to the residual. The values of $b$ and noise parameters are iterated until the noise fits within a pre-specified threshold. By this process, it is possible to minimise noise being interpreted as a signal. It also produces, using Student's t-test, measures of the confidence intervals of the resulting $b$ values by taking into account any covariance between the indices. (This model is developed by Myles Allen, University of Oxford). This methodology has been used in several climate studies, e.g., by Roy and Haigh (2010, 2012), Roy and Collins (2014) and Roy (2010, 2013).

The $\mathrm{Y}$ - variables used in this analysis are sea level pressure (SLP) and the $\mathrm{X}$-variables are the monthly SSN, geomagnetic aa index, Niño3.4 index, QBO, stratospheric aerosol optical depth (AOD) (indicative of volcanic eruptions) and a long-term trend. The analysis is mainly carried for boreal winter season, including the three consecutive months of the winter, December-January-February (DJF; January and February from the current year, December from the previous year). Before doing the regression, each predictor is normalised between -0.5 to +0.5 . Then the deviation of each year with respect to mean is calculated which is then divided by standard deviation of the same. Regression coefficients $(\mathrm{hPa})$ calculated here are thus given in terms of one standard deviation. Regression coefficients as derived for HadSLP data by Gray et al. (2013, Fig. 3) are also given in terms of the standard deviation of the predictors.

For SLP we use the HadSLP2 dataset from Allan et al. (2006), which covers the whole globe and is available as monthly means from 1850 to 2004 (http://www.metoffice. 
gov.uk/hadobs/hadslp2). It has been updated up to 2012 using HadSLP2r_lowvar data (http://www.metoffice.gov.uk/hadobs/hadslp2/data/ download.html), which is a version of HadSLP2r consistent with HadSLP2. The global monthly HadSLP2r dataset, covering 2005 to 2012, is the NCEP-NCAR Reanalysis data (Kistler et al., 2001). It is adjusted so that its average for period 1961-1990 matches with HadSLP2. The deficiency relating to difference in variance between HadSLP2 and HadSLP2r is adjusted in the new version of HadSLP2r_lowvar data. Monthly SSN is used to represent the solar cycle. http://www.ngdc.noaa.gov/stp/space-weather/solar-data/solar-indices/sunspotnumbers/international/listings/listing international -sunspot-numbers monthly.txt). The geomagnetic aa index is available since 1868 (ftp:// ftp.ngdc.noaa.gov/ STP/ GEOMAGNETIC DATA/AASTAR/aaindex). It is updated with the recent data from http://www.geomag.bgs.ac.uk/cgi-bin/aaindex. Monthly aa index shows a rising trend from December (solstice) to February (close to equinox), due to the well known semiannual variation of geomagnetic activity. In order to eliminate this variation the mean of each monthly series is first subtracted from the monthly indices of the respective month before doing the analysis. For ENSO we use the Niño 3.4 index (Kaplan et al., 1997) which is available since 1856 (http://climexp.knmi.nl). Global mean value of AOD has been used to represent volcanic eruptions (Sato et al. 1993), up to 1999 (http://data.giss.nasa. gov/modelforce/strataer/tau line.txt). It has been extended then to 2012 with near zero values. For QBO we use the reconstruction by Brönnimann et al. (2007) which extends from 1900 onwards (http://climexp.knmi.nl). Therefore we will limit our major analysis of regression to the time interval from 1900 until 2012.

The Pacific Decadal Oscillation (PDO) is a major variability originated in the mid-latitude of the Pacific. We also used PDO in place of ENSO in the regression to check the robustness of solar signal. However, it is noteworthy that the PDO is usually not considered as an independent variable, but rather a regional manifestation of longer term SST variability. Hence, we mainly restricted to ENSO, not to PDO, in the regression. The PDO dataset is available from http://iisao.washington.edu/ pdo/ for 1900-2005. There is no standard long-term record of TSI for a centennial analysis of climate. However, in the current analysis, we used the TSI reconstruction by Foster (2004). For these parameters also, the mean of each of the three months is first subtracted from the data of the respective month before doing the analysis.

Figure 1 shows the time series of all predictors used in this analysis.

\section{Results and Discussion}




\subsection{Differences and similarities of aa index and SSN on SLP during DJF}

The signals in DJF SLP pressure $(\mathrm{hPa})$ obtained from the multiple linear regression analysis are shown in Fig. 2. Top left panel shows the results for aa index, using a model including the aa index, AOD (volcano), Trend, ENSO and QBO (30hPa). Middle and bottom panels show signals due to four other factors (ENSO, AOD, Trend and QBO (30 $\mathrm{hPa}$ )). Similar analysis was also done for SSN (Fig. 2, top right panel) instead of aa index, including the same four other parameters (AOD, Trend, ENSO and QBO (30hPa)). Results for these four other factors (middle and bottom panels) produce a very similar pattern if we use SSN instead of aa index. The study using SSN is quite similar as Roy and Haigh (2010) but here the period from 1900 is considered instead of 1856, as we also used one additional term (QBO $(30 \mathrm{hPa}))$ in the regression. We tested the two common QBO heights of $30 \mathrm{hPa}$ and $50 \mathrm{hPa}$ in the regression. Although the patterns due to $\mathrm{QBO}(30)$ and $\mathrm{QBO}(50)$ somewhat differ, the solar signals remain quite similar with or without either of the QBO heights. (As seen in Fig. 2 the contribution of the QBO term remains rather weak overall). Here results using QBO $30 \mathrm{hPa}$ are presented.

The influence of the geomagnetic aa index to SLP is depicted in Fig. 2, top left panel. The strongest signatures are noticed in the central tropical Pacific, North Atlantic around Azores High pressure region and, especially in the northern polar regions, where a positive NAO/NAM type pattern in found, in agreement with earlier findings (Maliniemi et al., 2016). No significant signal is found around Aleutial Low (AL),

The most robust influence of SSN is seen around the AL (Fig. 2, top right), as earlier detected by Roy and Haigh (2010). It is also consistent with the studies of van Loon et al. (2007) who considered peak years of SSN cycle. Christofarao and Hameed (2007) also showed that $A L$ is weakened during active years of solar cycle. Apart from strong signature around $\mathrm{AL}$, a negative signal is also noticed around central tropical Pacific, though low in terms of amplitude but significant. This signal is quite similar to that in the aa index. Such negative signature can be responsible for inciting trade winds around central tropical Pacific and favouring initiation of the cold ENSO phase. Such indirect decadal dynamical coupling, observed here was discussed in detail by Roy (2013). It is in line with the observation by several studies of an underlying quasi-decadal variability in the inter-annual ENSO (White et al., 2008; Zhang et al., 1997; Zhao et al., 2003; Chen et al 2004). We also note that no signal is found around Azores High for sunspots. 
The signature of ENSO is found to be strongest around AL (Fig. 2, middle left). The signature of ENSO in AL is found to have opposite sign to that due to SSN activity. Volcanic activity (Fig. 2, middle right) indicates a positive NAO pattern. Trend (Fig. 2, bottom left) shows strong signatures in Greenland, AL and Antarctica. QBO (30) (Fig. 2, bottom right) shows mainly a negative anomaly in polar regions, similar to the aa index.

\subsubsection{Solar related signals around Aleutian Low (200 E, $60 \mathrm{~N})$ : aa index vs. SSN}

Figure 3 shows a SLP time series for a location at a place of the Aleutian Low (at location $160 \mathrm{~W}, 60 \mathrm{~N}$ ) for DJF during 1900 to 2004 . The coefficients of the components due to five explanatory factors $\left(\beta_{i} x_{i}, i=1\right.$ to 5$)$ are presented in the two lower panels and shown with five different colours. Middle panel uses aa index as the solar related index, while bottom panel considers SSN. The variance explained by these five factors for middle panel and bottom panels are both $97.9 \%$. As is seen, the ENSO captures major variability around AL. As to solar influence, the variability by SSN indicates a clearly stronger effect than the aa index. Extra-tropical forcing theory of ENSO (Kao and Yu 2009, Yu et al. 2010, Yu and Kim 2011) suggests that ENSO could be excited by extra-tropical forcing. AL could be one possible route for extra-tropical forcing of ENSO, but the effect could also be the other way round between the tropics and mid-latitudes of the Pacific (Mantu and Hare, 2002).

\subsubsection{Addressing on Solar ENSO behaviour based on SSN}

The effect of ENSO around AL matches with the effect of SSN (Fig. 2). Van Loon and Meehl (2007), using a longer period of data ( 150 years), detected a very strong tropical solar signal, resembling that of the negative phase of ENSO. To discuss their result a scatter plot is presented in Fig. 4 with ENSO during DJF vs. SSN, which is an updated plot from Roy and Haigh (2010). In Fig. 4, the additional solar maximum year of 2014 is also included. This analysis is based on peak (annual maximum) SSN years, as also used by van Loon et al (2007). Table 1 presents the sunspot numbers and the Niño 3.4 indices (DJF) during the peak years of SSN for the 15 solar cycles in 1856-2015.

No obvious relationship is seen between ENSO and SSN in Fig. 4, which is also confirmed (not shown here) by simple regression analysis using the two time series of SSN and ENSO (DJF). When, however, solar cycle maximum years are identified, ten of the fifteen have a value of ENSO index lower than the average (last peak year of 2014 inclusive), 
and three are equal to average. Only two peaks (cycles 14 and 16) show warm ENSO events, and these are peaks of rather weak solar cycles, with peaks below $\mathrm{SSN}=80$. Four of the sunspot maximum years $(1893,1917,1989,2000)$ are associated with particularly strong cold events (Table 1). As it is only the solar maximum years that are used by van Loon and Meehl (2007) to characterize the solar signal, it is clear that their result will resemble a cold event (La Niña) pattern. Apart from peak solar years, other years with high SSN also show a bias towards a cold event side of the ENSO. It is seen from Fig. 4 that more data points are towards cold event side when SSNs are high (say > SSN 80) and points are negatively skewed (skewness $=-0.98$ ). For SSN below 80 , data points are seen equally scattered with no such bias (skewness $=+0.54$ ). The negative solar signature for active and high SSN around central tropical Pacific that acts in a decadal time scale alongside usual interannual ENSO variability may be one responsible factor among others for indirect dynamical coupling, and needs more investigation. Considering the updated results, including the recent solar peak of 2014 which was again a cold event, it remains to be investigated to what extent the solar decadal variability can produce a surplus of cold events on inter-annual ENSO variability. (A similar analysis was repeated using aa index as a solar predictor, but did not indicate interesting biases and, hence, was not shown here).

\subsubsection{Seasonality, lag and robustness}

Figure 5 studies the seasonality of the ENSO response in the SLP variations around AL, It presents the pattern for ENSO in four different seasons: top panel uses DJF months (left) and March-April-May (MAM) (right); bottom panel shows the result using June-JulyAugust (JJA) (left) and September-October-November (SON) (right). It clearly shows that ENSO influence around $A L$ is seasonally dependent and strongest during boreal winter.

Fig. 6 shows results for the two solar related signals (top panels: aa index; bottom panels: SSN) using one year lag (left panels) and two year lag (right panels). A similar lag analysis was made by Gray et al (2013) using HadSLP2 dataset. In this study, however, we have included QBO as an additional predictor. For SSN, the signal around AL weakens below significance already at 1-year lag and disappears for 2-year lag. We also find that a signficant positive signal at 2-year lag around Azores High, in agreement with Gray et al. (2013). Interestingly, the negative response in the central tropical Pacific remains significant at least up to a 2-year lag. 
For aa index the NAO/NAM type signature at polar latitude disappears as a significant signal. However, the positive signal around Azores High is further strengthened at 1-year lag. It is interesting to note that the signal in the aa index around Azores High at 1-year lag is clearly stronger than that found for sunspots at any lag. For the aa index, this signal moves eastward for 2-year lag, covering most of Europe, with a center in the Baltic region $(20 \mathrm{E}, 60 \mathrm{~N})$. As another interesting finding, a weak negative signal appears around AL.

In order to check the robustness of the solar signal found using SSN around AL, we have also used TSI reconstruction by Foster (2004) instead of SSN. Figure 7 shows the results for TSI using no lag, 1-year lag and 2-year lag. We find that the signal around AL (at zero lag) is even stronger for TSI than SSN, and remains significant even un to 1-year lag, contrary to SSN. This gives additional support of a robust solar signal around AL. Moreover, the signal around Azores High behaves quite similarly for TSI as for SSN at all lags, but becomes significant in TSI already at 1-year lag.

In order to further study the robustness of SSN/TSI related signature around $A L$, a new predictor PDO was introduced instead of ENSO (see Fig. 7, bottom line), while keeping other indices the same as in Fig 2. We present the patterns here only for PDO and SSN. SSN still indicates a strong positive pattern around AL, whereas, the pattern of PDO suggests a negative response. The latter agrees with the sign of ENSO around AL during DJF (see Fig. 5).

\subsection{Combined influence of Solar and QBO on SLP during DJF: aa index vS SSN}

Baldwin and Dunkerton (2005) showed that fluctuations in the strength of stratospheric polar vortices are coupled downward with surface climate and speculated that the pathway involving the polar modes of variability appears to involve interactions of the solar effect with QBO. Here we used the product of solar and QBO (and termed as solar*QBO) to represent the collective behaviour involving both the solar and $\mathrm{QBO}$, as observed by Labitzke et al. (1992) and Labitzke (2004), who showed that warm polar temperatures tend to occur during the west phase of the QBO at solar maximum and east phase at solar minimum.

The combined solar ${ }^{\star}$ QBO index has also been used by Haigh and Roscoe $(2006 ; 2009)$ to investigate signals in polar modes and by Camargo and Sobel (2010) for tropical cyclones. Recent study of Roy and Haigh (2011) and Roy (2013) who applied this index 
to SLP, suggested that the effect of QBO combined with solar activity reveals a negative signal in the de-seasonalised NAM. In their MLR analyses they considered all months of the year and solar variability was represented by SSN. Their results indicated that high solar activity (HS) years during the westerly phase of the QBO and low solar activity (LS) years during the easterly phase of QBO, both trigger negative NAM features, supporting Labitzke's findings (1992, 2004).

QBO $30 \mathrm{hPa}$ in the combined index was found to give more clear and systematic results. Therefore, we present results only using that QBO height in Fig. 8. Results due to solar related variables as represented by aa index ${ }^{*} \mathrm{QBO}(30 \mathrm{hPa})$ and $\mathrm{SSN}^{*} \mathrm{QBO}(30 \mathrm{hPa})$ are shown in the top panels of Fig. 8. The signals for the other three indices of ENSO, AOD and Trend (for the aa index ${ }^{*}$ QBO model) are shown in the lower panels of Fig. 8. These results (middle and lower panel) are quite similar for the $S S N^{\star} \mathrm{QBO}$ model.

Neither of the combined indices indicate any significant response around AL. However, Figure 8 shows that aa index combined with QBO $(30 \mathrm{hPa})$ yields a strongly positive pattern (negative NAM) at polar latitudes, in accordance with Maliniemi et al. (2016). A strong significant positive pattern from $60 \mathrm{~N}$ to poleward is distinguished. There is a similar, but weaker pattern also for SSN combined with QBO, but its statistical significance is marginal.

This result indicates that low aa index and Easterly QBO are likely to cause high SLP from $60 \mathrm{~N}$ to pole during winter. This can cause a warm winter around North Pole if ENSO and volcanic influence can be neglected. This is also true during years with high aa and Westerly phase of QBO. On the other hand, cold Arctic winter is favoured when year of high (low) aa index ties with Easterly (Westerly) phase of QBO.

We check the temporal robustness of the NAM pattern for the combined aa index ${ }^{*}$ QBO using two different time periods, as depicted in Fig. 9. Top panel presents result for the first half (1900-1956) of the data record and the bottom panel for the second half (19572012). Both time intervals yield the same NAM pattern, although the signal is more significant in the second half. The robustness of the NAM pattern for the combined aa index ${ }^{*} \mathrm{QBO}$ could be very useful for future prediction of winter conditions at mid to polar latitudes in the northern hemisphere at seasonal to decadal time scales. It also has implications on location and strength of storm tracks in northern hemisphere during winter.

\section{Conclusion}


We have compared the effect of geomagnetic activity (as a proxy to magnetospheric energetic particles) and sunspot activity (as proxy to all sunspot related solar parameters like TSI and UV) to surface climate in northern winter using HadSLP2 dataset. Previous studies have only used SSN as the solar related predictor. Sunspot activity produces a strong positive influence around AL, which is further intensified by using TSI instead of sunspots. On the other hand, the aa index yields a positive NAM signal. Both of these results agree with the findings of earlier studies. There is a weak but significant negative signature present around central Pacific for both SSN and aa index. We also find a positive signal for the aa index around Azores High. Lag studies suggest that the positive signal around $\mathrm{AL}$ is stronger for TSI than SSN and valid up to 1-year lag for TSI. Interestingly, while there is a positive signal around Azores High for a 2-year lag in SSN, the strongest signal there is found for aa index at 1-year lag. Moreover, the combined influence of geomagnetic activity and QBO produces a very strong NAM response at mid to polar latitudes of the northern hemisphere. The signal is robust and irrespective of the time period chosen. This response is clearly stronger than that observed here or in previous studies when SSN is used as a solar related predictor in such a combination. Our results have a strong implication for winter weather prediction at middle to high latitudes of the northern hemisphere.

\section{Acknowledgement}

We acknowledge the financial support by the Academy of Finland to the ReSoLVE Centre of Excellence (project no. 272157). I. Roy also acknowledges support from ReCoVER project, UK. 


\section{References:}

Allan, R. and Ansell, T. (2006), A new globally complete monthly historical gridded mean sea level pressure dataset (HadSLP2): 1850-2004, J. Climate, 19(22), 5816-5842.

Baldwin, M.P. and Dunkerton, T.J. (2005), The solar cycle and stratosphere-troposphere dynamical coupling, J. Atmos. and Sol.-Terr. Phys., 67, 71-82, doi:10.1016/j.jastp.2004.07.018.

Barriopedro, D., R. Garcia-Herrera, and R. Huth (2008), Solar modulation of Northern Hemisphere winter blocking, J. Geophys. Res. 113, D14118, doi:10.1029/2008JD009789.

Baumgaertner, A. J. G., A. Seppälä, P. Jöckel, and M. A. Clilverd (2011), Geomagnetic activity related NOx enhancements and polar sur-face air temperature variability in a chemistry climate model: Modulation of the NAM index, Atmos. Chem. Phys., 11, 4521-4531, doi:10.5194/acp-11-4521-2011.

Bochnıcek, J., and P. Hejda (2005), The winter NAO pattern changes in association with solar and geomagnetic activity, J. Atmos. Sol. Terr. Phys., 67, 17-32, doi:10.1016/j.jastp.2004.07.014.

Bochnıcek, J., H. Davıdkovová, P. Hejda, and R. Huth (2012), Circulation changes in the winter lower atmosphere and long-lasting solar/geomagnetic activity, Ann. Geophys., 30, 1719-1726, doi:10.5194/angeo-30-1719-2012.

Brönnimann, S., J. L. Annis, C. Vogler, and P. D. Jones (2007), Reconstructing the quasibiennial oscillation back to the early 1900s, Geophys. Res. Lett., 34, L22805, 2007.

Camargo, S. J., and A. H. Sobel (2010), Revisiting the influence of the Quasi-Biennial Oscillation on tropical cyclone activity, J. Climate, 23, 5810-5825.

Chen, D., Cane, M. A., Kaplan, A., Zebiak, S. E. and Huang, D. J. (2004), Predictability of El Nino over the past 148 years. Nature, 428, 733736.

Christoforou, P. and Hameed, S. (1997), Solar cycle and the Pacific 'centers of action', Geophys. Res. Lett., 24(3), 293-296, doi: 10.1029/97GL00017.

Dima, M., Lohmann, G. and Dima, I. (2005): Solar-Induced and Internal Climate Variability at decadal time scales, International Journal of Climatology, 25, 713-733, doi:10.1002/joc.1156. 
Foster, S., (2004): Reconstruction of solar irradiance variations, for use in studies of global climate change: application of recent SoHO observations with historic data from the Greenwich Observatory, Ph.D. Thesis, University of Southampton, Southampton, U.K.

Funke, B., M. López-Puertas, S. Gil-López, T. von Clarmann, G. P. Stiller, H. Fischer, and S. Kellmann (2005), Downward transport of upper atmospheric NOx into the polar stratosphere and lower mesosphere during the Antarctic 2003 and Arctic 2002/2003 winters, J. Geophys. Res., 110, D24308, doi:10.1029/2005JD006463.

Gray, L.J., et al. (2010), Solar influences on climate. Rev. Geophys., 48, RG4001, doi:10. 1029/ 2009RG000282.

Gray, L.J., et al. (2013), A lagged response to the 11 year solar cycle in observed winter 612 Atlantic/ European weather patterns. Journal of Geophysical Research: Atmospheres, 118, 613 13,405-13,420, doi: 10.1002/2013JD020062.

Hegyi B. M., Deng Y. (2011), A dynamical fingerprint of tropical Pacific sea surface temperatures on the decadal-scale variability of cool season Arctic precipitation. J Geophys Res 116:D20. doi:10.1029/2011JD016001.

Haigh, J. D., Blackburn, M., and Day, R. (2005), The response of tropospheric circulation to perturbations in lower-stratospheric temperature, J. Climate, 18(17), 3672-3685.

Haigh, J.D. and Roscoe, H.K., (2006): Solar influences on polar modes of variability, Meteorol. Z., 15, 3, 371-378, doi: 10.1127/0941-2948/2006/0123.

Haigh, and Roscoe (2009). The final warming date of the Antarctic polar vortex and influences on its interannual variability. Journal of Climate, 22. 5809-5819. 10.1175/2009JCLI2865.1.

Ineson S. , Scaife A. A., Knight, J. R. et al. (2011), Solar forcing of winter climate variability in the Northern Hemisphere, Nature Geoscience, 753-757, DOI: 10.1038/NGEO1282.

Kao H-Y, Yu J-Y. (2009), Contrasting eastern-Pacific and central-Pacific types of El Nino. Journal of Climate, 22: 615 - 632.

Kistler, R., W. Collins, S. Saha, G. White, J. Woollen, E. Kalnay, M. Chelliah, W. Ebisuzaki, M. Kanamitsu, V. Kousky, H. van den Dool, R. Jenne, and M. Fiorino, 2001. The NCEP-NCAR 
50-year reanalysis: Monthly means, CD-ROM and documentation. Bulletin of the American Meteorological Society, 82: 247-267.

Kodera, K. and Kuroda, Y. (2002): Dynamical response to the solar cycle. J. Geophys. Res., 107, D24, 4749, doi:10.1029/2002JD002224.

Labitzke, K. and van Loon, H. (1992), On the association between the QBO and the extratropical stratosphere, J. Atmos. Terr. Phys., 54, 11/12, 1453-1463.

Labitzke, K. et al. (2004): On the Signal of the 11-Year Sunspot Cycle in the Stratosphere and its Modulation by the Quasi-Biennial Oscillation (QBO),J.A.S.T.P., special issue, $66,1151-1157$.

Lean, J. and Rind, D. (2001), Earth's response to a variable Sun, Science, 292, 5515, 234236.

Lockwood, M. et al. (2010), Are cold winters in Europe associated with low solar activity? Environ. Res. Lett., 5.

Li, Y., H. Lu, M. J. Jarvis, M. A. Clilverd, and B. Bates (2011), Nonlinear and nonstationary influences of geomagnetic activity on the winter North Atlantic Oscillation, J. Geophys. Res., 116, D16109, doi:10.1029/2011JD015822.

Maliniemi, V., T. Asikainen, K. Mursula, and A. Seppälä (2013), QBO-dependent relation between electron precipitation and wintertime surface temperature, J. Geophys. Res. Atmos., 118, doi:10.1002/jgrd.50518.

Maliniemi, V., T. Asikainen, and K. Mursula (2014), Spatial distribution of Northern Hemisphere winter tem-peratures during different phases of the solar cycle, J. Geophys. Res. Atmos., 119, doi:10.1002/2013JD021343.

Mantua, N.J. and Hare, S. R. (2002): The Pacific Decadal Oscillation. Journal of Oceanography, 58, 1, 35-44.

McPhaden MJ, Lee T, McClurg D. (2011). El Niño and its relationship to changing background conditions in the tropical Pacific Ocean. Geophysical Research Letters. 38: L15709, DOI: 10.1029/2011GL048275

Meehl, G. A., Arblaster, J.M., Branstator, G., and van Loon, H. (2008), A coupled air-sea response mechanism to solar forcing in the Pacific region, J. Climate, 21(12), 2883-2897. 
Meehl, G. A. and Arblaster, J. M. (2009): A Lagged Warm Event-Like Response to Peaks in Solar Forcing in the Pacific Region, J. Climate., 22(13), 3647-3660.

Rozanov, E., L. Callis, M. Schlesinger, F. Yang, N. Andronova, and V. Zubov (2005), Atmospheric response to NOy source due to energetic electron precipitation, Geophys. Res. Lett., 32, L14811, doi:10.1029/2005GL023041.

Roy, I. and Haigh, J. D. (2010) Solar cycle signals in sea level pressure and sea surface temperature, Atmos. Chem. Phys., 10, 6, 3147-3153.

Roy, I. (2010) Solar Cycle signals in Sea Level Pressure and Sea Surface Temperature, Ph.D. Thesis, Department of Space and atmospheric Science, Imperial College London.

Roy, I. and Haigh, J.D. (2011), The influence of solar variability and the quasi-biennial oscillation on lower atmospheric temperatures and sea level pressure, Atmos. Chem. Phys., 11, 11679-11687, doi: 10.5194/acp-11-11679-2011.

Roy, I. and Haigh, J.D. (2012), Solar Cycle Signals in the Pacific and the Issue of Timings. Journal of Atmospheric Science, 69, 4, 1446-1451, doi: http://dx.doi.org/10.1175/JAS-D-110277.1.

Roy, I., (2013), The role of the sun in atmosphere-ocean coupling International Journal of Climatology, doi:10.1002/joc.3713.

Roy and Collins, (2014) On identifying the role of Sun and the El Niño Southern Oscillation on Indian Summer Monsoon Rainfall, Atmos. Sci. Let. DOI: 10.1002/asI2.547

Sato, M., Hansen, J. E., McCormick, M. P. and Pollack, J. B. (1993), Stratospheric aerosol optical depths (1850 - 1990), J. Geophys. Res., 98, 22, 987 -22, 994.

Seppälä, A., P. T. Verronen, M. A. Clilverd, C. E. Randall, J. Tamminen, V. Sofieva, L. Backman, and E. Kyrölä (2007), Arctic and Antarctic polar winter NOx and energetic particle precipitation in 2002-2006, Geophys. Res. Lett., 34, L12810, doi:10.1029/2007GL029733.

Seppälä, A., C. E. Randall, M. A. Clilverd, E. Rozanov, and C. J. Rodger (2009), Geomagnetic activity and polar surface air temperature variability, J. Geophys. Res., 114, A10312, doi:10.1029/2008JA014029. 
Seppälä, A., H. Lu, M. A. Clilverd, and C. J. Rodger (2013), Geomagnetic activity signatures in wintertime stratosphere wind, temperature, and wave response, J. Geophys. Res. Atmos., 118, 2169-2183, doi:10.1002/jgrd.50236.

Sinnhuber, M., S. Kazeminejad, and J. M. Wissing (2011), Interannual variation of NOx from the lower thermosphere to the upper stratosphere in the years 1991-2005, J. Geophys. Res., 116, A02312, doi:10.1029/2010JA015825.

Smith, T.M. and Reynolds, R. M. (2004): Improved extended reconstruction of SST (18541997). J. Climate, 17, 2466-2477.

Thejll, P., B. Christiansen, and H. Gleisner (2003), On correlations between the North Atlantic oscillation, geopotential heights, and geomagnetic activity, Geophys. Res. Lett., 30(6), 1347, doi:10.1029/2002GL016598.

Tung, K. K., and J. S. Zhou, (2010), Solar cycle in 150 years of global sea-surface temperature, J. of Atm. Sciences, 67, 2649-2657.

van Loon, H., Meehl, G.A., and Arblaster, J.M., (2004): A decadal solar effect in the tropics in July-August, J. Atmos. Sol.-Terr. Phy., 66(18), 1767-1778.

van Loon, H., Meehl, G.A., and Shea, D.J. (2007), Coupled air-sea response to solar forcing in the Pacific region during northern winter, J Geophys. Res.-Atmos., 112, D02108, doi:10.1029/2006JD007378.

White, W. B. and Liu, Z. Y., (2008): Non-linear alignment of El Nino to the 11-yr solar cycle, Geophys. Res. Lett., 35, L19607, doi:10.1029/2008GL034831.

Woollings, T., M. Lockwood, G. Masato, C. Bell, and L. Gray (2010), Enhanced signature of solar variability in Eurasian winter climate, Geophys. Res. Lett., 37, L20805, doi:10.1029/2010GL044601.

Yu., J.-Y., H.-Y. Kao and T. Lee, (2010), Subtropics-Related Interannual Sea Surface Temperature Variability in the Equatorial Central Pacific. Journal of Climate, 23, 2869-2884

Yu., J.-Y. and S. T. Kim, (2011), Relationships between Extratropical Sea Level Pressure Variations and the Central-Pacific and Eastern-Pacific Types of ENSO, Journal of Climate, 24, 708-720. 
Zhang, Y., Wallace, J. M. and Battisti, D. S. (1997), ENSO-like interdecadal variability: 190093. J. Climate, 10, 1004-1020.

Zhao, M. and Dirmeyer, P. (2003), Pattern and trend analysis of temperature in a set of seasonal ensemble simulations. Geophys. Res. Lett., 30, doi:10.1029/2003GL018579. 
Table 1: Niño 3.4 index (DJF) at the peak year of SSN for the 15 solar cycles 1856-2015. There are three years with Niño 3.4 index (DJF) close to zero (within $-0.02{ }^{\circ} \mathrm{C}$ to $0.02{ }^{\circ} \mathrm{C}$ ), two positive and ten negative Niño 3.4 index years.

\begin{tabular}{|c|c|c|c|c|}
\hline Solar Cycle No & Year & Peak year & SSN (annual average) & ENSO(DJF) \\
\hline 10 & $1856-1867$ & 1860 & 95.8 & -0.44 \\
\hline 11 & $1867-1878$ & 1870 & 138.9 & -0.82 \\
\hline 12 & $1878-1889$ & 1883 & 63.6 & -0.36 \\
\hline 13 & $1889-1901$ & 1893 & 85.1 & -1.43 \\
\hline 14 & $1901-1913$ & 1905 & 63.5 & 1.24 \\
\hline 15 & $1913-1923$ & 1917 & 103.9 & -1.33 \\
\hline 16 & $1923-1933$ & 1928 & 77.8 & 0.14 \\
\hline 17 & $1934-1944$ & 1937 & 114.4 & -0.01 \\
\hline 18 & 1944-1954 & 1947 & 151.5 & .013 \\
\hline 19 & $1955-1964$ & 1957 & 189.8 & -0.15 \\
\hline 20 & 1964-1976 & 1968 & 105.9 & -0.64 \\
\hline 21 & $1976-1986$ & 1979 & 155.3 & 0.02 \\
\hline 22 & $1986-1996$ & 1989 & 157.8 & -1.83 \\
\hline 23 & $1996-2007$ & 2000 & 119.5 & -1.65 \\
\hline 24 & $2008-2015$ & 2014 & 79.08 & -0.37 \\
\hline Total & Solar cycle & & & \\
\hline
\end{tabular}



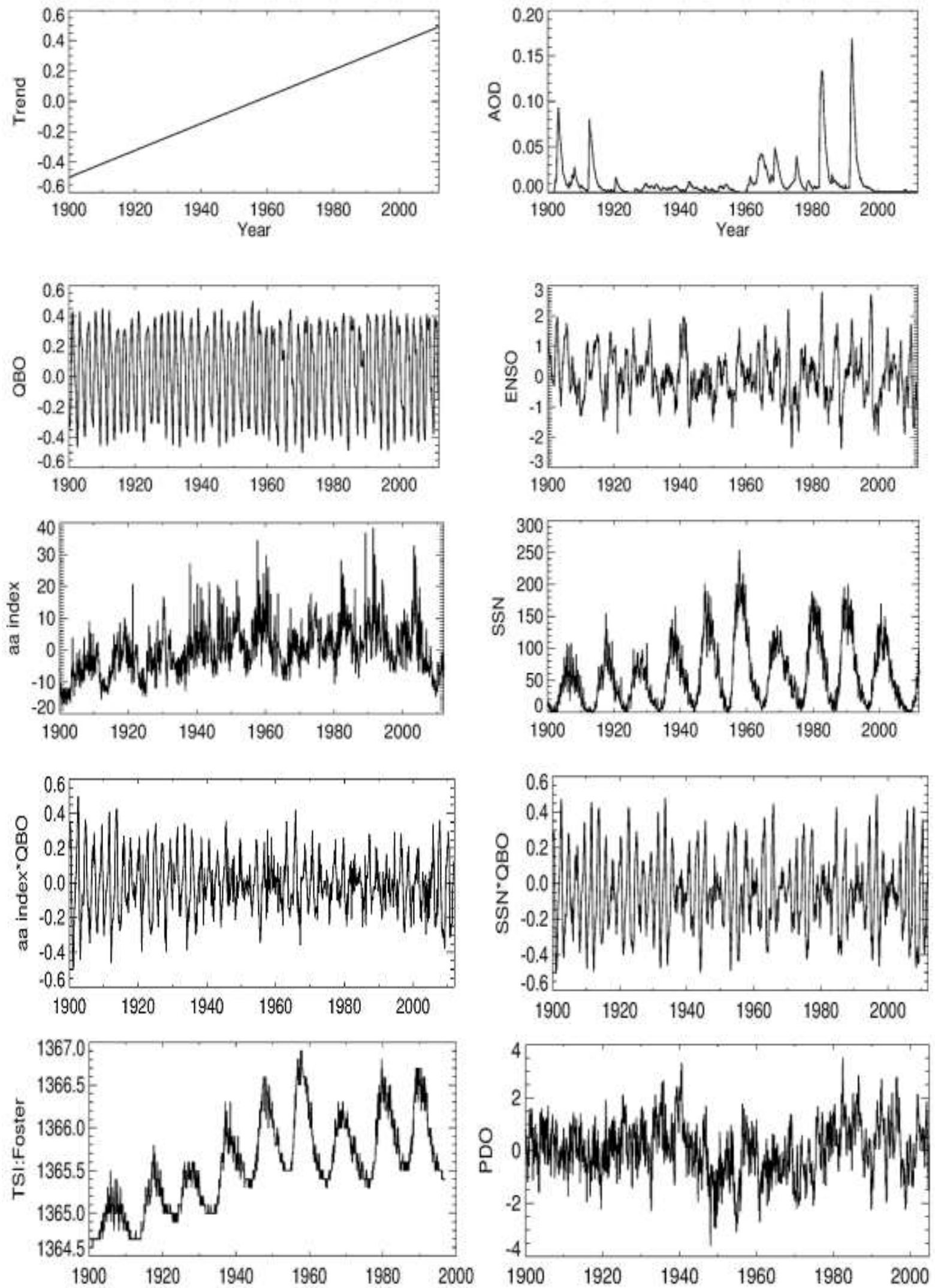

Fig.1. Time series of the indices used in the analysis. 
Solar(aa index

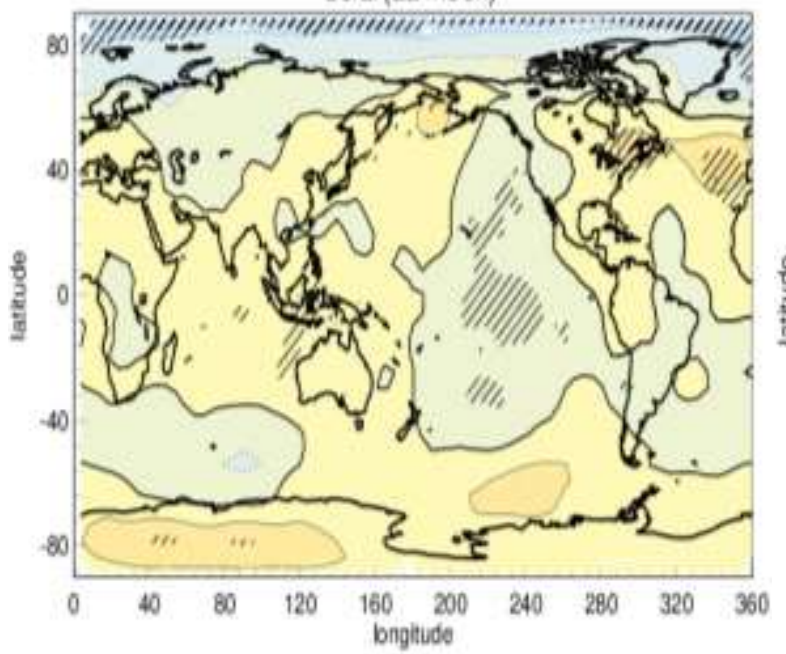

ENSO

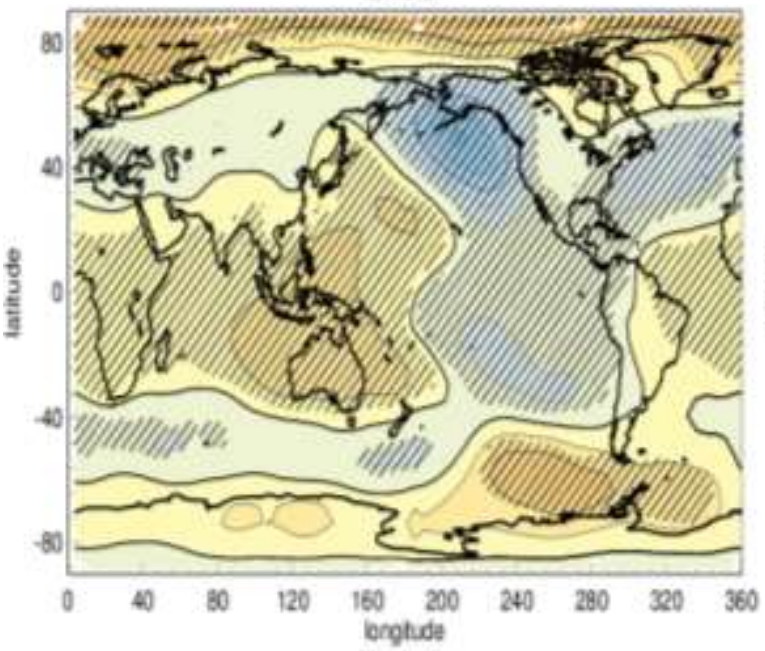

Trend

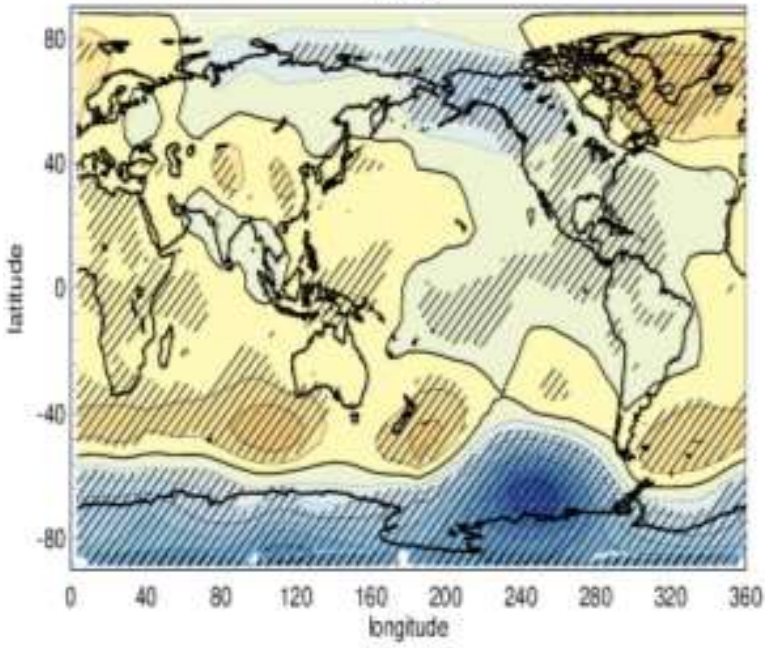

$\begin{array}{lllllllllll}-3.0 & 2.4 & 1.8 & -12 & -0.6 & 0.0 & 0.6 & 1.2 & 1.8 & 2.4 & >30\end{array}$
Solar(SSN)
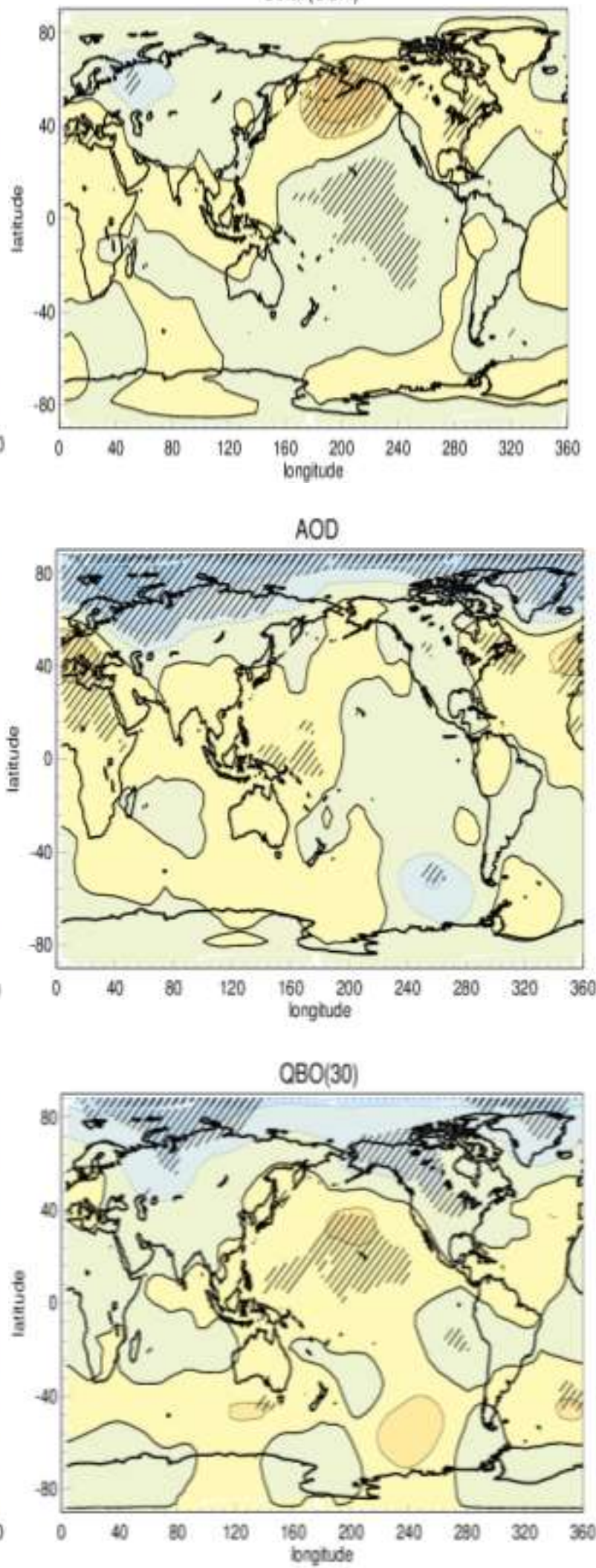

$\begin{array}{lllllllllll}-3.0 & -2.4 & -1.8 & +12 & -0.5 & 0.0 & 0.5 & 1.2 & 1.8 & 2.4 & >3.0\end{array}$ 
Fig.2. The signal (max-min, hPa) in DJF, Hadley centre SLP data, obtained from a multiple linear regression analysis using various indices over the 1900-2012 period, measured in terms of standard deviation. Results due to solar cycle variability as represented by aa index or SSN are shown in top panels. Using aa index, the signals for other indices, ENSO, AOD (volcano), QBO (30hPa) and trend are shown in lower panels. Negative contours are shown by dotted lines and zero lines are marked by thick line. Shaded regions are estimated significant at the $95 \%$ level using a two-sided Student's $t$ test. 

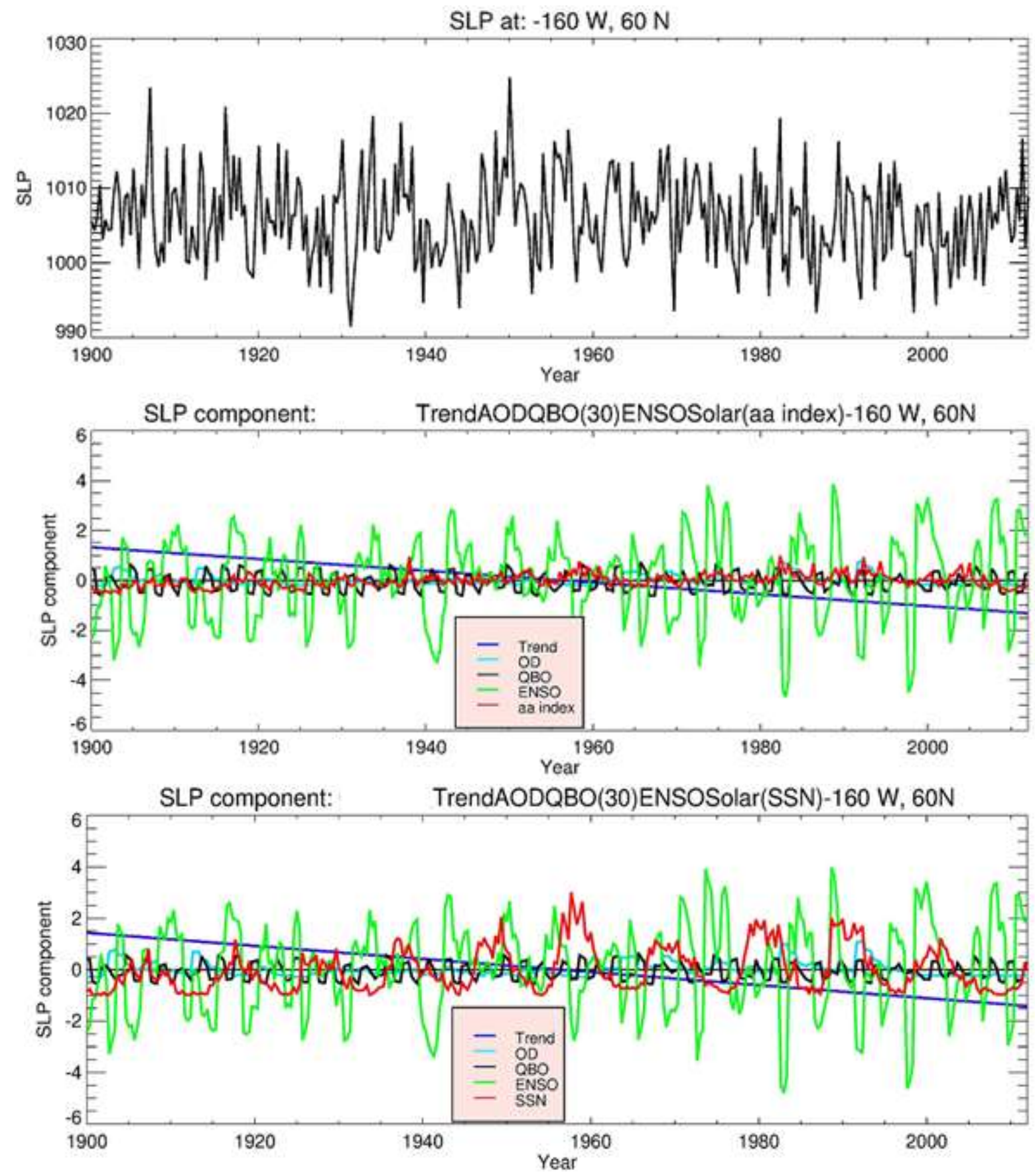

Fig. 3. Variability of SLP (hPa) around Aleutian Low (at location 160W, 60N) for DJF during 1900 to 2012 (top) and amplitudes of the components of variability due to Trend (blue), AOD (cyan), QBO (black), ENSO (green) and solar activity (red) (bottom). Middle row uses aa index as solar activity and bottom row SSN. 


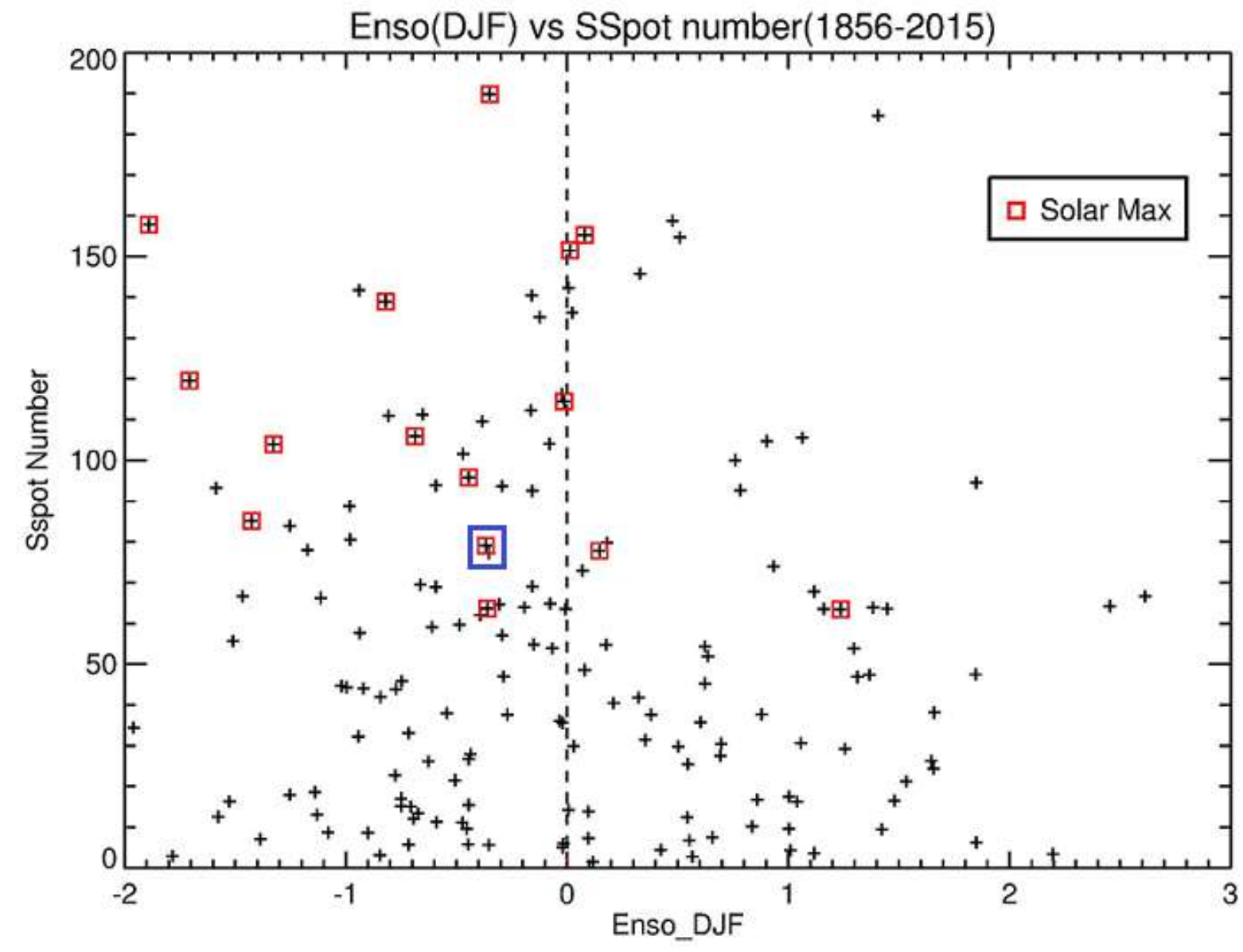

Fig.4. Scatter plot of ENSO index (DJF) values against annual average sunspot number from 1856 to 2015 inclusive. A symbol surrounded by a red square indicates the peak year of a solar cycle. The recent peak year 2014 is marked by additional blue square. 

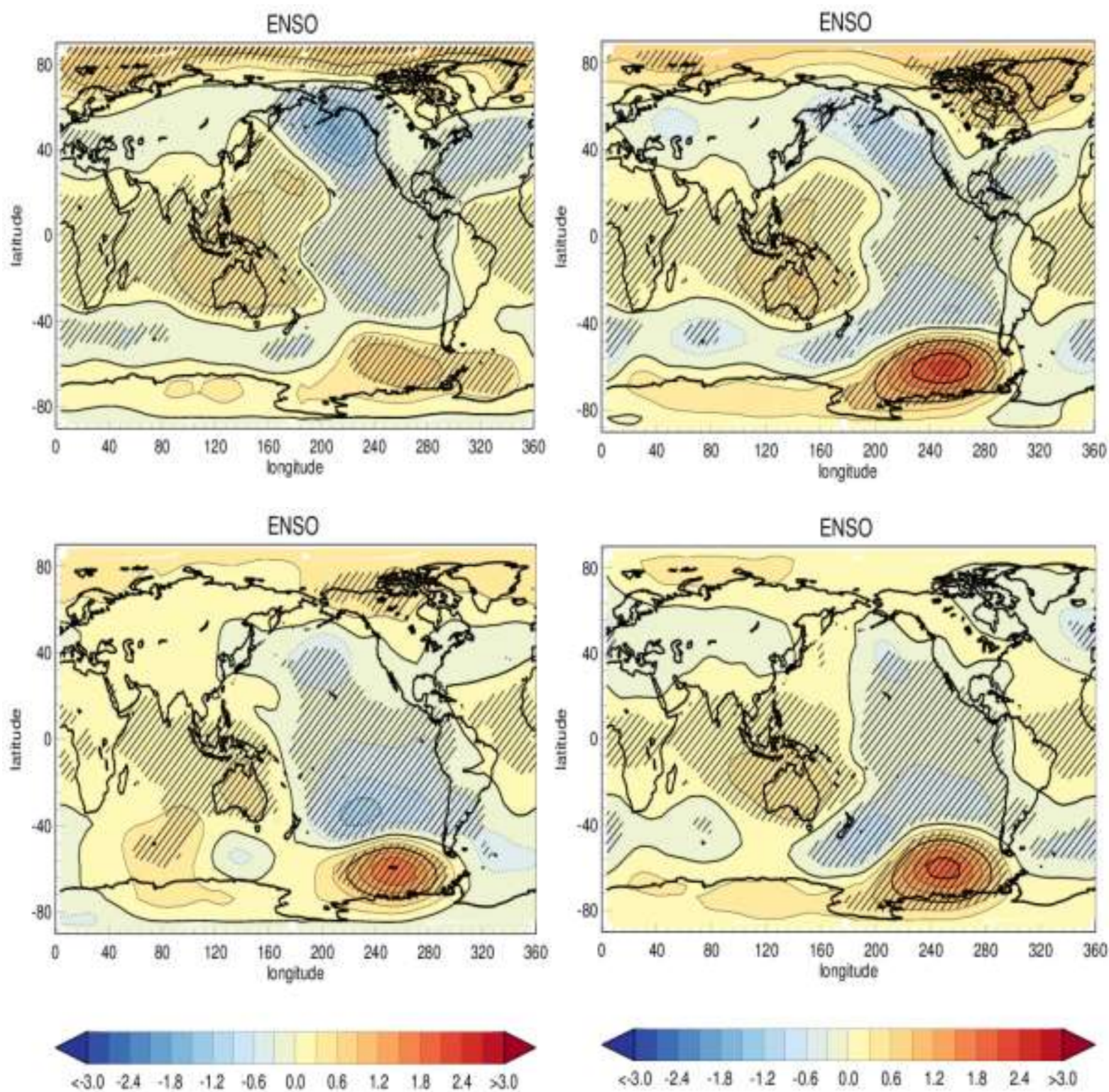

Fig.5. Same as Fig 2, but only for ENSO. Top panel uses months DJF (left) and MAM (right); whereas, bottom panel shows results only using months JJA (left) and SON (right). 

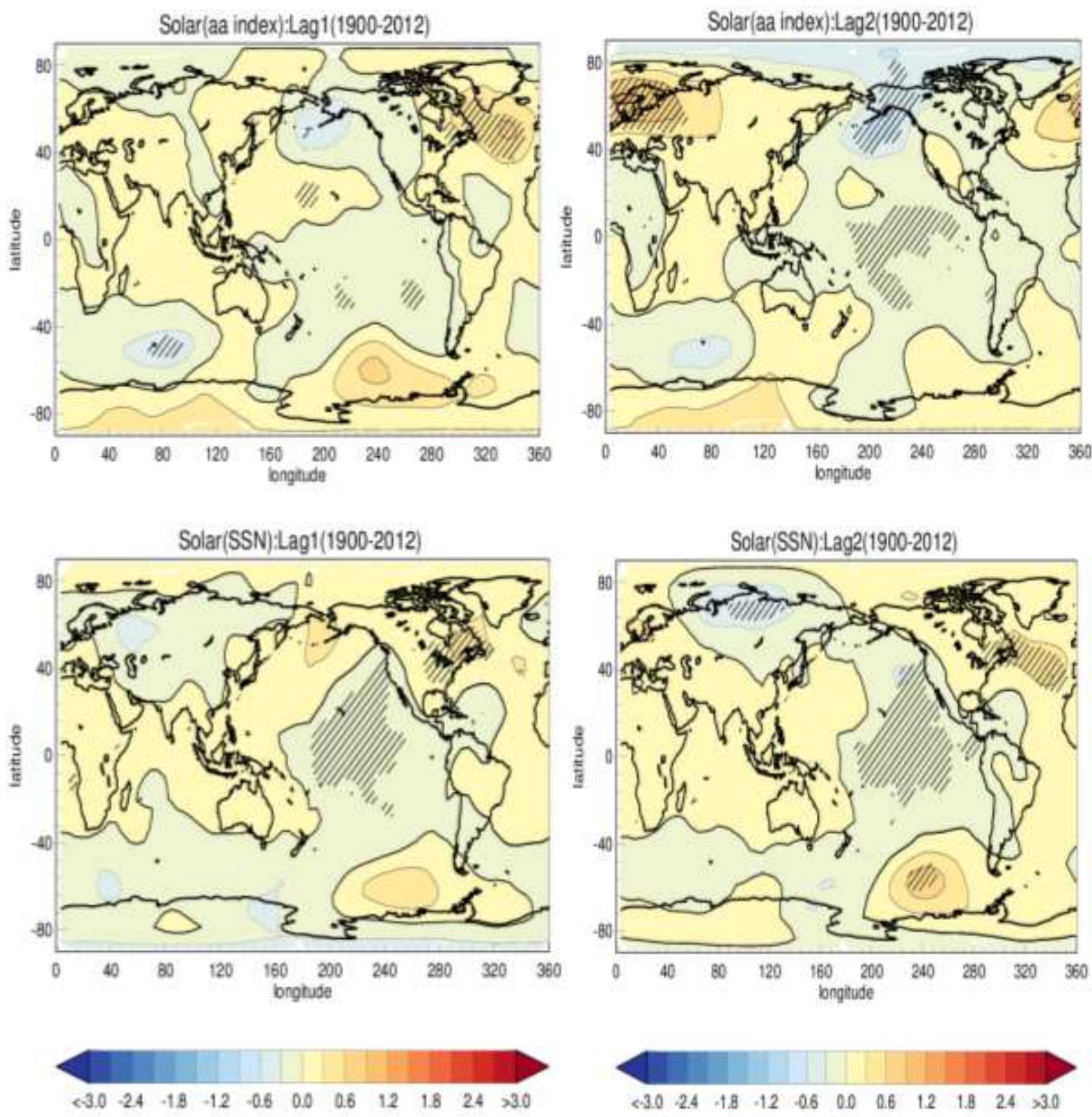

Fig. 6. Same as Fig. 2 but for solar signals using one year lag (left panel) and two year lag (right panel). Top panel is using aa index and bottom panel for SSN. 

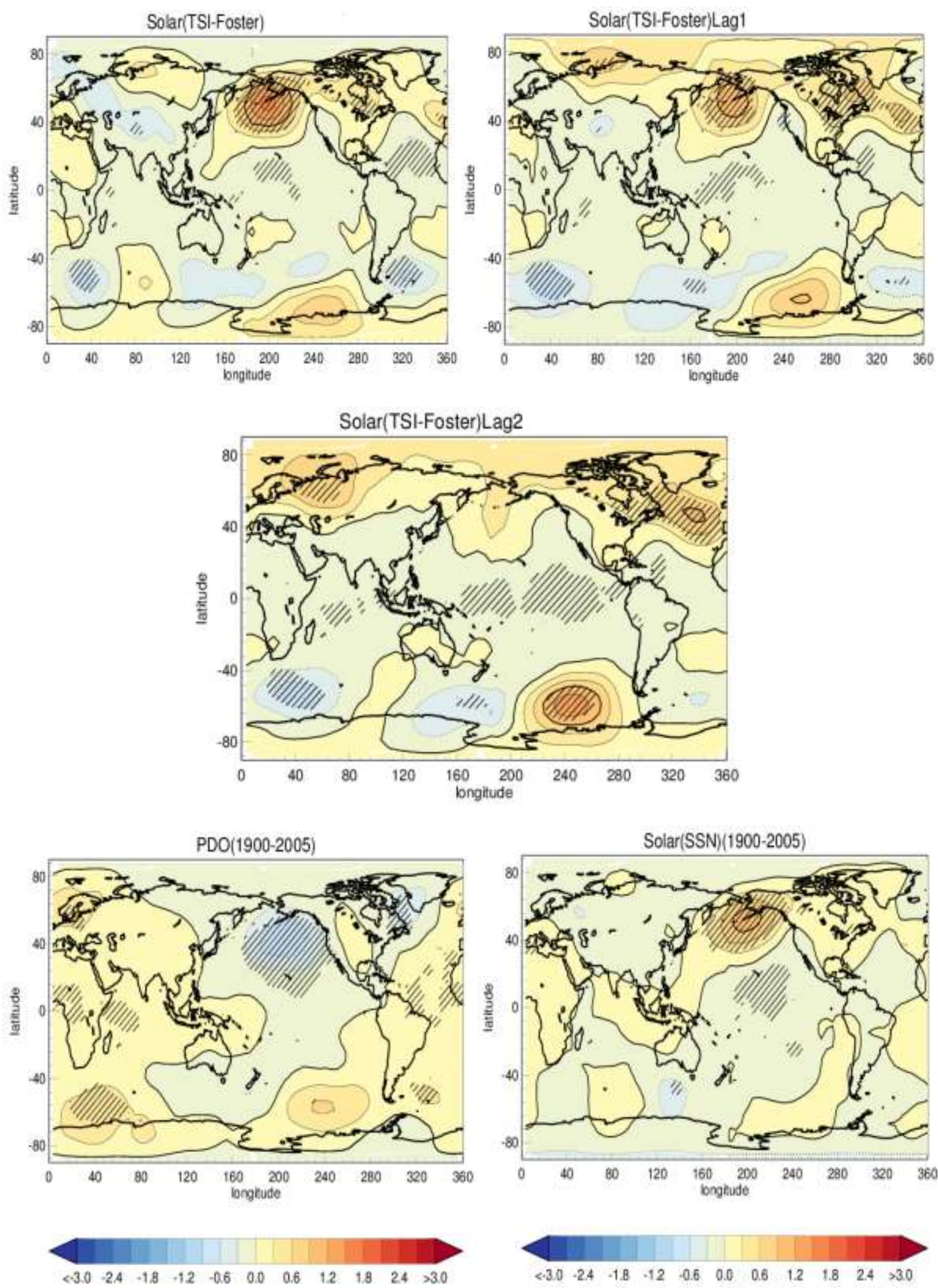

Fig. 7. Same as Fig 2, but using TSI as solar index at zero lag (top left) at 1-year lag (top right) and at 2-year lag (middle panel). Bottom panels use PDO instead of ENSO with other indices the same as in Fig. 2. Results presented are only for PDO (left) and SSN (right). 
aa index $X \mathrm{OBO}(30)$

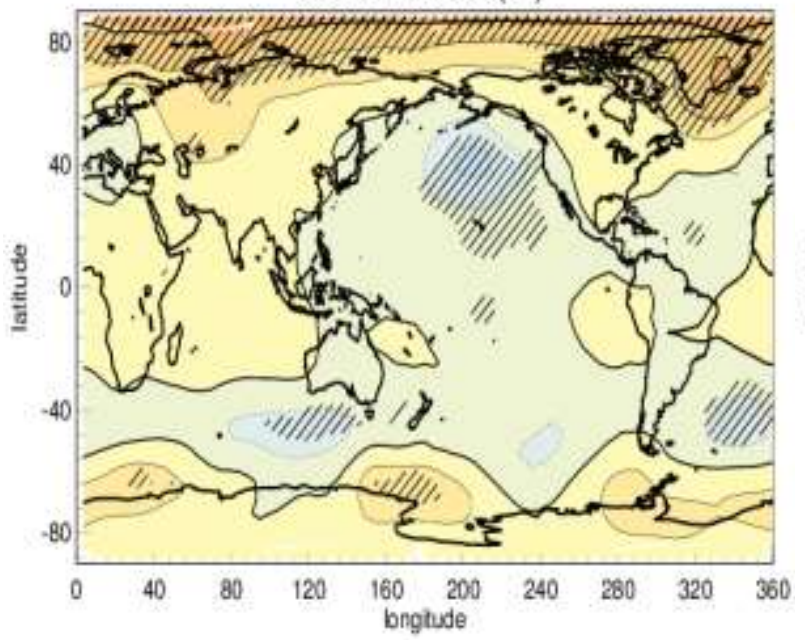

SSN XQBO(30)

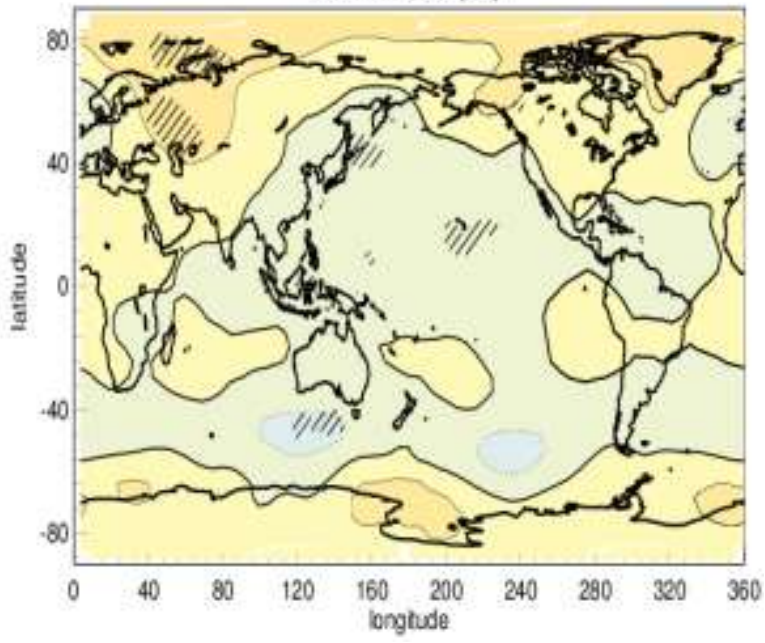

ENSO

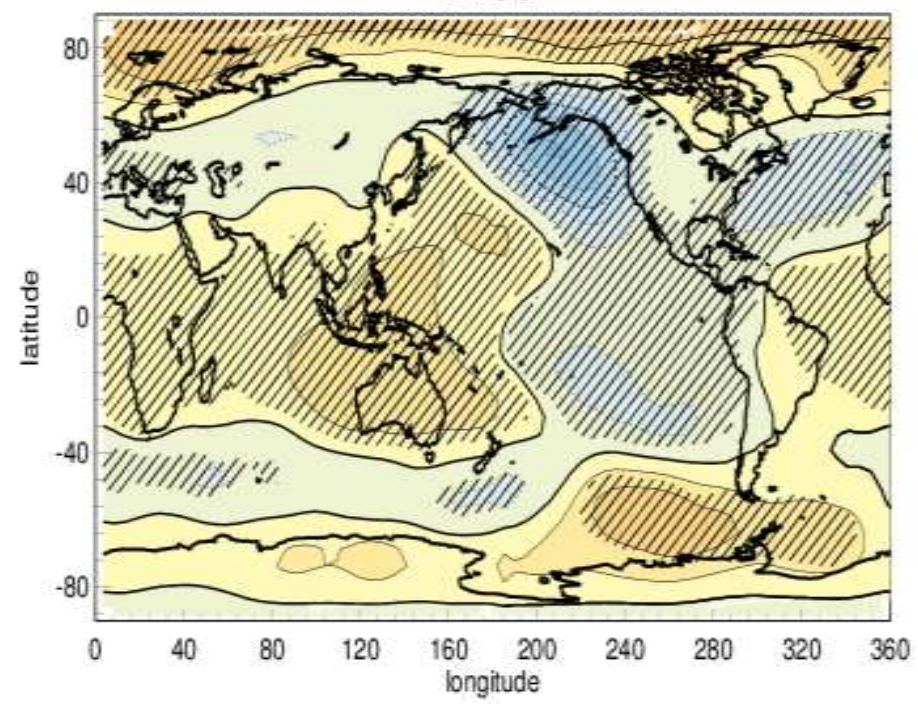

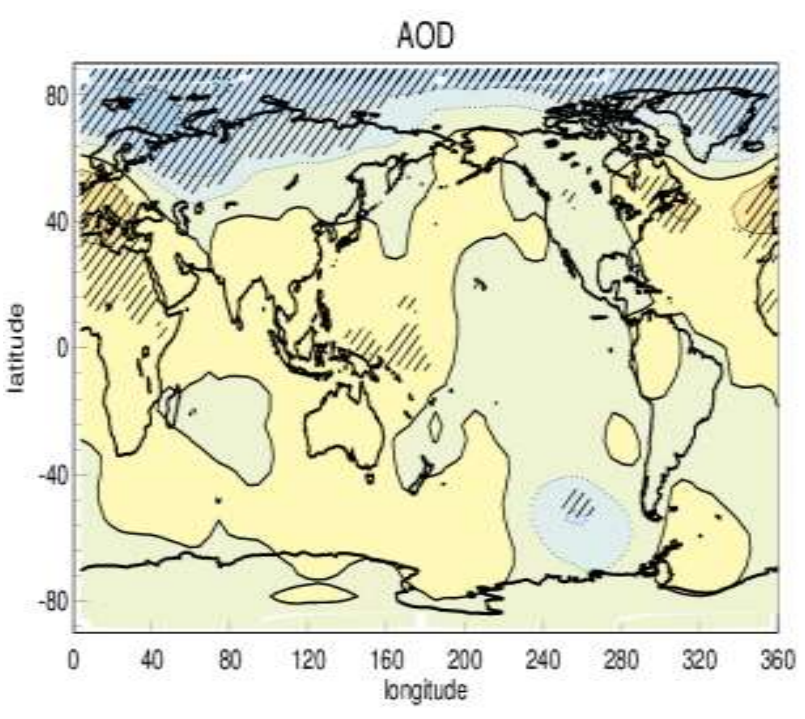

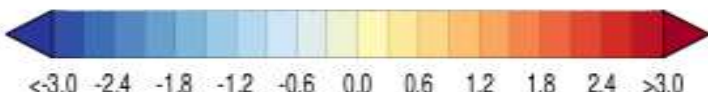

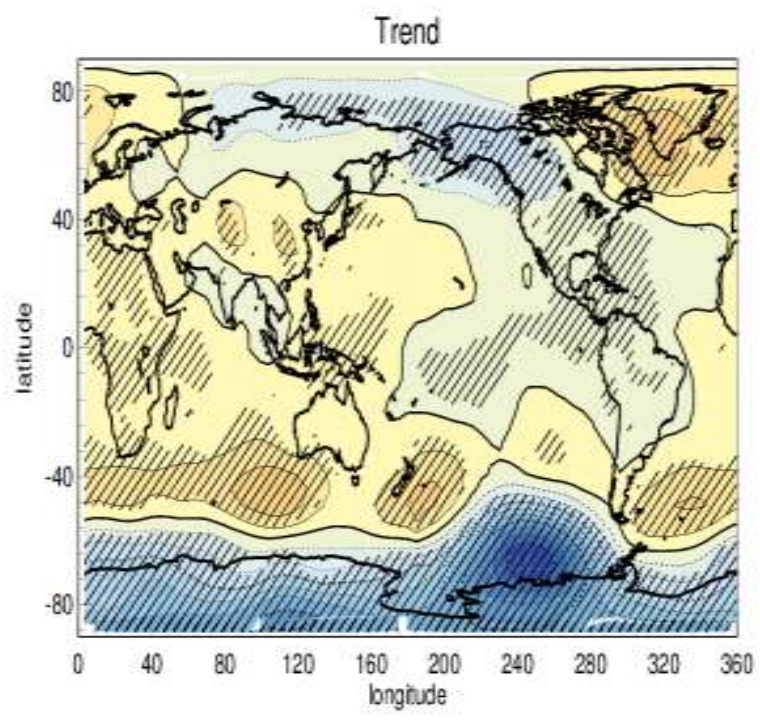

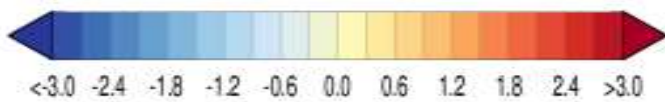


Fig. 8. Same as Figure 2, but the combination $\mathrm{Solar}^{\star} \mathrm{QBO}(30 \mathrm{hPa})$ is used as solar related variable and shown in top panels. Results for aa index ${ }^{*} \mathrm{QBO}$ is in top left, and for $\mathrm{SSN}^{*} \mathrm{QBO}$ in top right. Other signals shown in lower panels are due to ENSO, AOD (volcano) and trend using aa index ${ }^{\star} \mathrm{QBO}$ as one regressor. Similar results are obtained using SSN instead of aa index. 

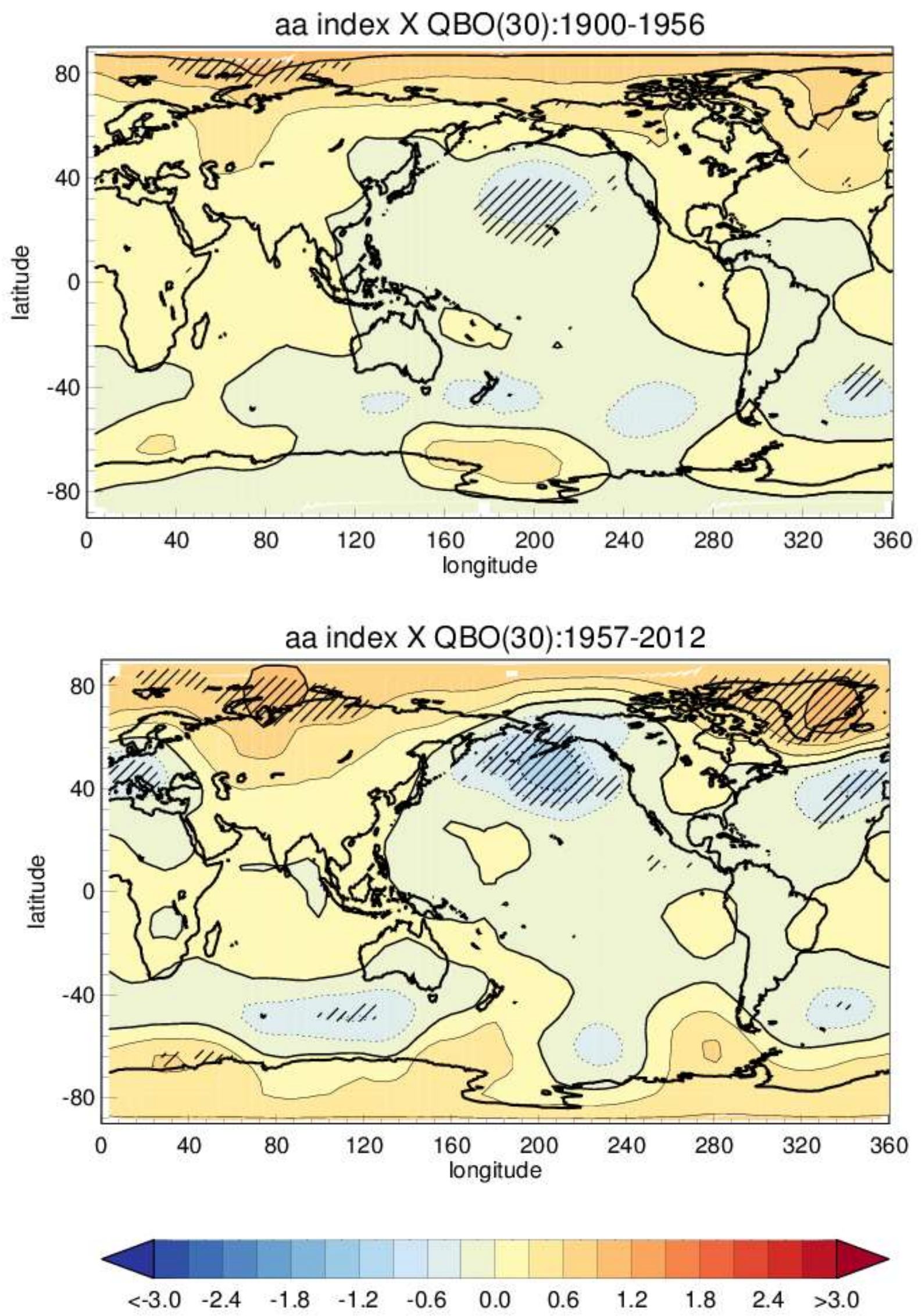

Fig. 9. Same as Fig 8, but for aa index ${ }^{*} \mathrm{QBO}(30)$ in two different periods. Top panel is for 1900-1956 and the bottom panel for 1957-2012. 